\section{In vitro fertilisation and stage 3 retinopathy of prematurity}

significance $(p=0.35$ and $p=0.13$, respectively).

Conclusions In this study $11.7 \%$ of the group requiring screening were conceived by assisted conception. Of all babies requiring treatment for ROP, $28.6 \%$ were born after assisted conception. Of the assisted conception group, $83.3 \%$ were conceived by IVF. Assisted conception using IVF rather than other techniques appears to be the major risk factor for the development of threshold ROP. We would advise increased vigilance when screening babies conceived by the IVF methods of assisted conception.

Key words In vitro fertilisation, Prematurity, Retinopathy

During 1996 and 1997, 25565 patients received in vitro fertilisation (IVF) treatment, which resulted in 5601 live birth events. ${ }^{1}$ In 1993 McFaul et al. ${ }^{2}$ reported that $8 \%$ of babies born from assisted conception were born with a birth weight of $1500 \mathrm{~g}$ or less, compared with $1 \%$ of those conceived naturally. ${ }^{3}$ These infants would fulfil the criteria set out by the Royal College of Ophthalmologists and British Association of Perinatal Medicine for screening for retinopathy of prematurity (ROP). ${ }^{4}$ In 1996 McKibbin and Dabbs $^{5}$ examined the workload imposed by treatment for infertility on the ROP screening programme and found that $16.5 \%$ of babies screened were born after assisted conception. They reported that, of the assisted conception infants, 23\% developed some stage of ROP and $25 \%$ required treatment. However, they did not differentiate between the different forms of assisted conception and the development of retinopathy.

We had also noticed an increased workload with babies born after assisted conception and felt that the method of conception influenced the development and severity of ROP. We therefore identified those infants presenting to a neonatal intensive care unit in North West London who were conceived through assisted conception and observed the percentage of those infants developing acute ROP and their method of conception. 


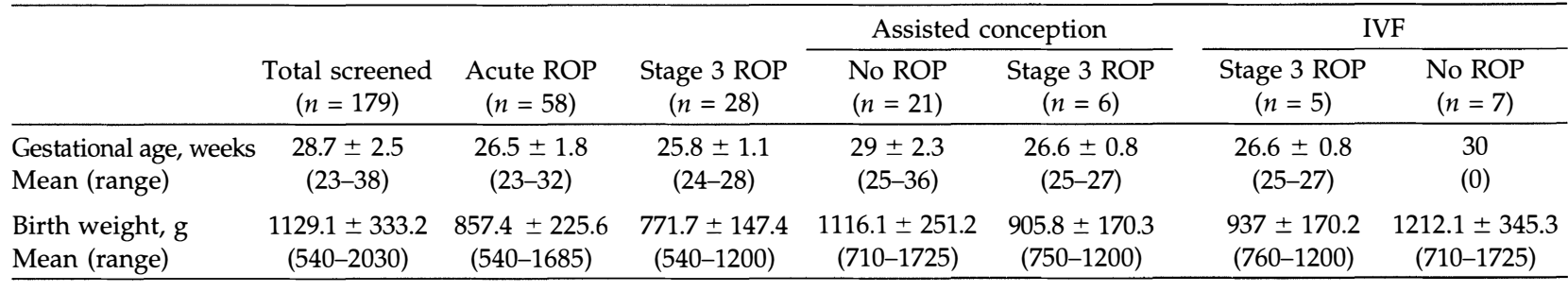

\section{Patients and methods}

Between December 1995 and December 1998 the number of infants who fulfilled the Royal College of Ophthalmologists and British Association of Perinatal Medicine criteria for screening for ROP were identified from the ROP screening database. The infant's neonatal case notes and mother's obstetric case notes were reviewed.

The details of the type of assisted conception, the birth history, the gestational age and birth weight details were recorded. The presence of singleton or multiple pregnancies and the details of the fundal examination were recorded.

The types of assisted conception utilised were IVF (which includes in vitro fertilisation without micromanipulation (IVF) and the new technique of intracytoplasmic sperm injection (ICSI)), intrauterine insemination (IUI) and the use of clomiphene. Unless otherwise stated the term IVF includes techniques with and without micromanipulation.

Statistical analysis was carried out using Microsoft Excel 97 for descriptive statistics and the SPSS software for significance testing.

\section{Results}

One hundred and seventy-nine infants met the screening criteria for ROP during the study period. The mean gestational age was $28.79 \pm 2.51$ weeks (range 23-38 weeks) and the birth weight $1129.15 \pm 333.23 \mathrm{~g}$ (range 540-2030 g). Fifty-eight infants (32.4\%) developed some stage of acute ROP. The gestational age and birth weight of the 58 patients who developed acute ROP were $26.55 \pm 1.82$ weeks (range 23-32 weeks) and $857.41 \pm 225.65 \mathrm{~g}$ (range 540-1685 g). The data are given in Table 1.

Twenty-eight of these infants (15.6\%) developed acute stage 3 ROP. There were 16 females and 12 males. Sixteen infants were Caucasian, 9 were Asian and 3 were Afro-Caribbean.

There were 21 infants conceived by assisted means. Twelve were conceived by IVF (7 with IVF without micromanipulation and 5 by ICSI), 1 by IUI and 8 with the help of clomiphene (Table 2). Acute ROP of any stage developed in 7 infants of the assisted fertilisation group; 1 reached stage 1 and 6 infants reached stage 3 ROP. One of these infants was conceived on clomiphene and 5 with IVF ( 3 with ICSI and 2 with IVF without micromanipulation). The latter 5 IVF infants all required treatment for threshold disease. Though the numbers were small there was a trend for the IVF infants with stage 3 ROP to have a higher gestational age and birth weight than the rest of the infants with stage 3 disease, but this did not approach statistical significance ( $p=0.35$ and $p=0.13$, respectively) (Table 3 ).

There were 26 multiple pregnancies of which 23 were twin gestations and 3 were triplets. Eleven ( 1 from a triplet pregnancy and 10 from twin pregnancies) of the infants from multiple pregnancies died before screening for ROP could be undertaken. This left 44 infants $(24.58 \%)$ from multiple gestations. Of the 21 infants conceived through assisted conception, 14 were from multiple gestations. Seven infants from multiple pregnancies $(15.9 \%)$ reached stage 3 ROP, of which 5 were conceived through IVF treatment.

\section{Discussion}

The human fertilisation and embryology authority (HFEA) has licensed 114 clinics in the UK to carry out treatment and research in the various forms of assisted conception. ${ }^{1}$ The assisted conception techniques offered consist of IVF (which unless otherwise stated includes other micromanipulation techniques of ICSI and subzonal insemination, or SUZI), gamete intrafallopian tube transfer (GIFT), donor insemination (DI), intrauterine insemination (IUI) and the use of gonadotrophins. In our study 5 babies were born after ICSI treatment, 7 after IVF without micromanipulation treatment, 1 after IUI and 8 after treatment with clomiphene.

Table 2. Distribution of assisted conceptions and stage of ROP reached

\begin{tabular}{lccc}
\hline $\begin{array}{l}\text { Type of assisted } \\
\text { conception }\end{array}$ & $\begin{array}{c}\text { No. of } \\
\text { infants }\end{array}$ & $\begin{array}{c}\text { No. with } \\
\text { ROP }\end{array}$ & $\begin{array}{c}\text { No. with stage 3 } \\
\text { ROP }\end{array}$ \\
\hline IVF & 7 & 2 & 2 \\
ICSI & 5 & 3 & 3 \\
IUI & 1 & 0 & 0 \\
Clomiphene & 8 & 2 & 1 \\
\hline
\end{tabular}

IVF, in vitro fertilisation without micromanipulation; ICSI, intracytoplasmic sperm injection; IUI, intrauterine insemination. As ICSI is a form of IVF, for data analysis it is considered together with IVF without micromanipulation. 
Table 3. Birth weight and gestational age in infants reaching stage 3 ROP: IVF versus other assisted conception techniques

\begin{tabular}{lccccc}
\hline & No. of infants & Sex & Race & Birth weight $(\mathrm{g})$ & Gestational age (weeks) \\
\hline $\begin{array}{l}\text { Stage 3 ROP - not IVF } \\
\text { (natural + clomiphene) }\end{array}$ & 23 & $14 \mathrm{~F}$ & $3 \mathrm{AC}$ & $735.7 \pm 117.7$ & $25.7 \pm 1.1$ \\
& & $9 \mathrm{M}$ & $7 \mathrm{~A}$ & & $26.6 \pm 0.89$ \\
Stage 3 ROP - IVF & 5 & $2 \mathrm{~F}$ & 13C & $937 \pm 170.2$ & $2 \mathrm{C}$ \\
& & $3 \mathrm{M}$ & $1 \mathrm{~A}$ & & \\
\hline
\end{tabular}

F, female; M, male; AC, Afro-Caribbean; A, Asian, C, Caucasian.

As a result of the activity of various units offering assisted conception, the outcome of these pregnancies has seen an increase in the number of premature infants ( $24 \%$ for assisted conceptions compared with $6 \%$ of those naturally conceived) and low birth weight infants (32\% conceived by assisted conception compared with 7\% conceived naturally). ${ }^{3}$ This is largely due to the high frequency of multiple births in assisted conceptions compared with natural conceptions ( $23 \%$ vs $1 \%) .{ }^{3}$ About $8 \%$ of assisted conception babies, compared with $1 \%$ of those conceived naturally, weigh less than $1500 \mathrm{~g}^{2}$ and hence would merit screening for ROP. This will underestimate the number of assisted conception babies requiring screening as it does not include those born before 32 weeks who weigh more than $1500 \mathrm{~g}$. In 1996 McKibbin and Dabbs ${ }^{5}$ reported that $16.5 \%$ of infants who fulfilled the ROP screening criteria were born after assisted conception. Screening data were available on 29 of these infants. Ten infants developed ROP of any stage, and 3 infants progressed to stage 3 , of whom 2 required treatment for threshold disease. The mode of assisted conception for these infants was not recorded. Our data concur with McKibbin and Dabbs' findings in some aspects. Of their cohort $29.6 \%$ had any stage of ROP, whilst we found $32.4 \%$ in our series. Twenty-three per cent of their assisted conception cohort developed some form of retinopathy whilst the figure was $33.3 \%$ in our series. Twenty-five per cent of their series requiring treatment were born after assisted conception compared with $28.6 \%$ in our cohort.

In our series $11.7 \%$ of the infants screened for ROP were born after assisted conceptions. Of these infants $33.3 \%$ (7 infants) developed some stage of ROP, of whom $87.5 \%$ (6 infants) reached stage 3 , and $71.4 \%$ (5 infants) required treatment for threshold disease. The remaining infant did not progress beyond stage 1 ROP.

The IVF infants accounted for $57.1 \%$ (12 infants) of assisted conceptions, with $41.6 \%$ progressing to stage 3 threshold disease compared with $9.37 \%$ of those born naturally. All IVF infants who developed any stage of ROP progressed to threshold disease. Those IVF infants with ROP had a lower gestational age (26.6 \pm 0.89 weeks) and birth weight $(937 \pm 170.2 \mathrm{~g})$ than those IVF infants who did not develop ROP (gestational age $30.25 \pm 0.7$ weeks, birth weight $1206.87 \pm 320.04 \mathrm{~g}$ ). Of the assisted conception babies needing treatment, $83.3 \%$ were born after IVF treatment.

Amongst the 179 infants in our series, $29.5 \%$ of the infants born of multiple gestations had some stage of ROP, with $13.6 \%$ reaching stage 1 and $15.9 \%$ reaching stage 3 ROP. Some stage of ROP was present in $33.3 \%$ of singleton pregnancies; stage 1 ROP was present in $12.5 \%$, stage 2 ROP in $5.18 \%$ and stage 3 in $15.5 \%$. These percentages represent the maximum stages of ROP reached before either regression was noted or treatment initiated.

The development of ROP in infants conceived from assisted conceptions is largely a result of these infants being born prematurely with very low birth weights and low gestational ages. This is to a large extent related to the high frequency of multiple pregnancies associated with assisted conceptions. Blumenfeld et al. ${ }^{6}$ found in a large series that there was no difference in the incidence or the severity of ROP between singleton and multiple gestation babies. Although the number of infants conceived through IVF treatment compared with those conceived naturally is small, multiple pregnancies alone do not account for the increased percentage of infants reaching threshold disease in this group. The difference in outcomes of assisted conception has been postulated to be due to the fact that the gametes were exposed to a variety of drugs, physically manipulated, nurtured in potentially hazardous conditions, and perhaps placed in an inappropriate uterine environment. ${ }^{2}$ This, in addition to immaturity, may have some relevance in the development of their eye disease. It certainly appears that infants conceived with the aid of clomiphene, a gonadotrophin which causes ovarian stimulation, have a lower risk of developing threshold ROP.

\section{Conclusions}

Our data support previous suggestions that babies born after assisted conception have a higher incidence of acute ROP. This is particularly so for the children conceived by IVF. It appears that IVF-assisted conception is the major risk factor rather than gonadotrophin stimulation. We would advise greater vigilance when screening these very precious babies conceived by IVF as they appear to be at greater risk of developing acute sight-threatening retinopathy. This association of IVF and ROP has not been previously documented.

The authors wish to thank Catey Bunce (Medical Statistician, Glaxo Department of Epidemiology, Moorfields Eye Hospital), Rachel Abraham and Ann Tubritt (Clinical Audit Department) and Lis Temple (Neonatal Unit, Northwick Park Hospital) for their help in this study. 


\section{References}

1. Human Fertilisation and Embryology Authority. Seventh annual report. Collecting and providing data: IVF data. London: Human Fertilisation and Embryology Authority, 1998: 5-21.

2. McFaul PB, Patel N, Mills J. An audit of the obstetric outcome of 148 consecutive pregnancies from assisted conception: implications for neonatal services. Br J Obstet Gynaecol 1993;100:820-5.

3. MRC working party on children conceived by in vitro fertilisation. Birth in Great Britain resulting from assisted conception, 1978-87. BMJ 1990;300:1229-33.
4. Royal College of Ophthalmologists and British Association of Perinatal Medicine. Retinopathy of prematurity: guidelines for screening and treatment. The report of a joint working party. London: RCO/BAPM, 1995.

5. McKibbin M, Dabbs TR. Assisted conception and retinopathy of prematurity. Eye 1996;10:476-8.

6. Blumenfeld LC, Siatkowski RM, Johnson, Feuer WJ, Flynn JT. Retinopathy of prematurity in multiple-gestation pregnancies. Am J Ophthalmol 1998;125:197-203. 